\title{
Author Correction: Chemosynthetic symbionts of marine invertebrate animals are capable of nitrogen fixation
}

Jillian M. Petersen, Anna Kemper, Harald Gruber-Vodicka, Ulisse Cardini, Matthijs van der Geest, Manuel Kleiner, Silvia Bulgheresi, Marc Mußmann, Craig Herbold, Brandon K. B. Seah, Chakkiath Paul Antony, Dan Liu, Alexandra Belitz and Miriam Weber

Correction to: Nature Microbiology https://doi.org/10.1038/nmicrobiol.2016.195, published online 24 October 2016.

In this Article, the completeness and number of contigs for draft genomes from two individuals of Laxus oneistus are incorrect in the main text, although the correct information is included in Table 1. The original and corrected versions of the relevant sentence are shown in the correction notice.

Original sentence:

Those from two individuals of L. oneistus ( $\mathrm{Ca}$. Thiosymbion oneisti A-B) were 86.75 and $89.11 \%$ complete at sizes of 3.66 and $3.51 \mathrm{Mb}$ on 183 and 193 contigs (Table 1 ).

Corrected sentence:

Those from two individuals of $L$. oneistus ( $\mathrm{Ca}$. Thiosymbion oneisti A-B) were 96.63 and $96.07 \%$ complete at sizes of 4.44 and $4.33 \mathrm{Mb}$ on 2,026 and 1,891 contigs (Table 1 ). 